\title{
A sensitive technique by differential scanning for detecting haemoglobins of fetal origin
}

\author{
M. C. OWEN \\ From the Department of Clinical Biochemistry, Christchurch Hospital, Christchurch, New Zealand
}

SYNOPSIS A simple modification is made to the ultraviolet scanning technique for the detection of the tryptophan notch which is characteristic of haemoglobins of fetalorigin. This involves a differential scan using $\mathrm{Hb}$ A solution in the reference compartment. This technique has all the advantages of the standard scan method but extends the scope of it by being much more sensitive and decisive

It is often necessary to determine whether a haemoglobin sample or an abnormal band is of fetal origin with the presence of gamma chains. Examples are the identification of haemoglobin Barts on electrophoresis, the differentiation of an abnormal fetal or adult band in cord blood, and the identification of blood from a haemorrhage as being either of fetal or maternal origin. The routine techniques are either staining the red cells for fetal haemoglobin after acid elution of adult haemoglobin (Kleihauer) or an alkali denaturation. Other techniques include a spectral scan to detect the tryptophan notch at about $290 \mathrm{~nm}$, and an amino acid analysis of the hydrolysed protein to determine the presence of isoleucine which is peculiar to gamma but not alpha, beta, or delta chains. The ultraviolet scan is to be preferred when there is only a small amount of relatively dilute sample. This technique is very rapid, sensitive, and does not destroy the sample. Its chief disadvantage is that in many cases an unequivocal result is not obtained. A simple improvement is reported which greatly increases the discriminatory power of the method. This involves doing a differential scan using as the reference sample $\mathrm{Hb}$ A of similar concentration of the test specimen.

\section{Methods and Results}

The haemoglobin A reference solution should be in the same buffer or solvent as the unknown and be of a similar concentration. Adjustment of the more concentrated solution to a concentration equal to the more dilute solution can usually be done quite satisfactorily by visual comparison. Where the

Received for publication 10 October 1974 unknown solution is a peak from a column separa tion, the reference solution would simply be one ob the other known peaks, $\mathrm{Hb} \mathrm{A}$ or $\mathrm{Hb} \mathrm{A_{2 }}$, from ther same run. If, however, the unknown is a band from haemoglobin electrophoresis it would be eluted by standard techniques with the electrophoretic buffere and the reference solution would similarly be कo known band eluted under the same conditions $\frac{}{\circ}$ Where a barbitone buffer or one that absorbs under? certain conditions in the ultraviolet spectral region? is used extra care must be taken to ensure that the sample and reference solvents are identical. In isolated cases it may be necessary to dialyse both solutions against the same buffer or solvent.

The scan can be performed either on a twin beamo ultraviolet spectrophotometer or manually on single beam instrument measuring every $2 \mathrm{~nm} 8$ between about $275 \mathrm{~nm}$ and $300 \mathrm{~nm}$. In both cases the unknown solution is in the sample compartment ande the $\mathrm{Hb}$ A solution in the referenc e position.

A scan is shown (fig 1) of $\mathrm{Hb}$ A against distilled water, $\mathrm{Hb} \mathrm{F}$ against distilled wat er, and $\mathrm{Hb} \mathrm{F}$ agains $\mathrm{Hb} \mathrm{A}$. At best $\mathrm{Hb} F$ gives a scan with a change in slope from negative to positive and back to negative giving a maximum at about $290 \mathrm{~nm}$ and a minimum N at about $288 \mathrm{~nm}$. However, in practice the result iso usually an inflexion or shoulder at about $290 \mathrm{~nm}^{\circ}$ which is neither a true $\mathrm{Hb} \mathrm{A}$ nor a true $\mathrm{Hb} \mathrm{F}_{\mathbb{D}}^{\mathrm{C}}$ spectrum. The differential scan of $\mathrm{Hb} \mathrm{F}$, in contrast $\stackrel{\oplus}{\rightarrow}$ gives a distinct peak with the baseline on either sideo being almost flat. It also enables an unequivoca $\vec{P}$ result from samples which are considerably more

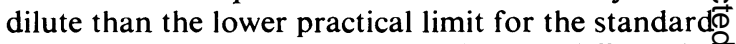
scan technique. The peak obtained with a differentia $E_{-}$ scan on $\mathrm{Hb}$ Barts, as would be expected, is even more pronounced than $\mathrm{Hb} \mathrm{F}$. When the unknown 


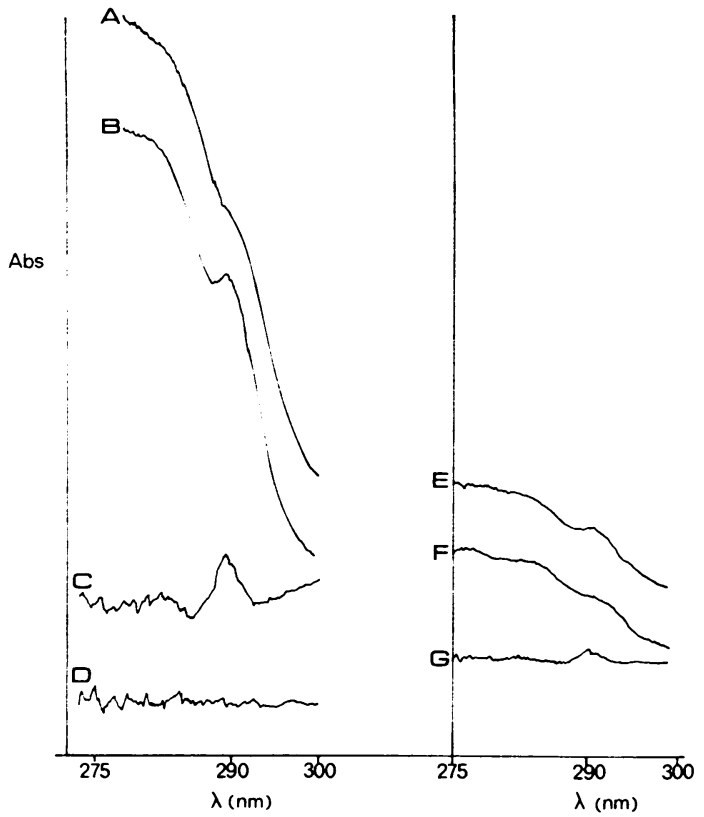

(a)

sample is $\mathrm{Hb} \mathrm{A}$ a straight line is obtained with a differential scan.

\section{Discussion}

Ager and Lehmann (1958) found that Hb Barts had the ultraviolet tryptophan notch that was first reported for Hb F by Jope (1949) and subsequently thought to be characteristic for Hb F. This spectral scan technique has since been employed by many laboratories. Although this method can be used to detect fetal haemoglobin in a whole haemolysate, it is mainly used to confirm rapidly or to exclude the presence of gamma chains in either a band from electrophoresis or a peak from column chromatography. However, on occasion this proves incapable of giving a decisive result, particularly with dilute solutions. An unknown band from a cord blood sample is often only $10 \%$ or less of the total haemoglobin. Indeed some fetal variants (Huisman, Schroeder, Bannister, and Grech, 1972) constitute only about $5 \%$ of the total fetal haemoglobin in the cord blood. It is in cases such as these that a rapid and reliable classification is needed as to whether a band is a fetal fraction or an adult fraction. An early answer can avoid both time being wasted pursuing false leads and interesting fetal variants being missed.

This work was supported in part by the NZ Medical Research Council.

\section{References}

Ager, J. A. M., and Lehmann, H. (1958). Observations on some 'fast' haemoglobins: K, J, N, and 'Barts'. Brit. med. J., 929-931.

Huisman, T. H. J., Schroeder, W. A., Bannister, W. H., and Grech, J. L. (1972). Evidence for four nonallelic structural genes for the $\gamma$ chain of human fetal haemoglobin. Biochem. Genet., 7, 131-139.

Jope, E. M. (1949). Hasmoglobin, pp. 205-219. Butterworth, London. 